Review Article

\title{
Herbal Medicines for Treating Metabolic Syndrome: A Systematic Review of Randomized Controlled Trials
}

\author{
Soobin Jang, ${ }^{1}$ Bo-Hyoung Jang, ${ }^{2}$ Youme Ko, ${ }^{1}$ Yui Sasaki, ${ }^{1}$ Jeong-Su Park, ${ }^{3}$ \\ Eui-Hyoung Hwang, ${ }^{4}$ Yun-Kyung Song, ${ }^{5}$ Yong-Cheol Shin, ${ }^{2}$ and Seong-Gyu Ko ${ }^{2}$ \\ ${ }^{1}$ Department of Korean Preventive Medicine, Graduate School, Kyung Hee University, Seoul 02447, Republic of Korea \\ ${ }^{2}$ Department of Preventive Medicine, College of Korean Medicine, Kyung Hee University, Seoul 02447, Republic of Korea \\ ${ }^{3}$ Department of Healthcare Safety Research, National Evidence-Based Healthcare Collaborating Agency, \\ Seoul 04554, Republic of Korea \\ ${ }^{4}$ Third Division of Clinical Medicine, School of Korean Medicine, Pusan National University, Yangsan 50612, Republic of Korea \\ ${ }^{5}$ Department of Korean Medicine Rehabilitation, College of Korean Medicine, Gachon University, Incheon 21565, Republic of Korea
}

Correspondence should be addressed to Yun-Kyung Song; oxyzen@korea.com and Seong-Gyu Ko; epiko@khu.ac.kr

Received 19 January 2016; Accepted 11 May 2016

Academic Editor: Chris Zaslawski

Copyright (C) 2016 Soobin Jang et al. This is an open access article distributed under the Creative Commons Attribution License, which permits unrestricted use, distribution, and reproduction in any medium, provided the original work is properly cited.

Objective. The aim of this systematic review is to evaluate the efficacy and safety of herbal medicines in the management of metabolic syndrome. Materials and Methods. On December 9, 2015, we searched PubMed, EMBASE, Cochrane Library, SCOPUS, AMED, CNKI, KoreaMed, KMBASE, OASIS, and J-STAGE with no restriction on language or published year. We selected randomized controlled trials that involved patients with metabolic syndrome being treated with herbal medicines as intervention. The main keywords were "Chinese herbal medicines", "metabolic syndrome", and "randomized controlled trials". Herbal substances which were not based on East Asian medical theory, combination therapy with western medicines, and concurrent diseases other than metabolic syndrome were excluded. The risk of bias was assessed by Cochrane's "Risk of Bias" tool. The protocol or review was registered in PROSPERO (an international prospective register of systematic reviews) (CRD42014006842). Results. From 1,098 articles, 12 RCTs were included in this review: five trials studied herbal medicines versus a placebo or no treatment, and seven trials studied herbal medicines versus western medicines. Herbal medicines were effective on decreasing waist circumference, blood glucose, blood lipids, and blood pressure. Conclusion. This study suggests the possibility that herbal medicines can be complementary and alternative medicines for metabolic syndrome.

\section{Background}

Metabolic syndrome is a complex disorder consisting of central obesity, hyperglycemia, hypertension, and hyperlipidemia [1]. There have been different diagnosis criteria for metabolic syndrome after it was first being defined by the World Health Organization (WHO) in 1998 [2]. The most commonly accepted definition uses the criteria suggested by the American Heart Association/National Heart Lung and Blood Institute (AHA/NHLBI).

According to the AHA/NHLBI, metabolic syndrome is technically diagnosed when three or more of the following five conditions are met: (1) waist circumference (WC) $\geq 102 \mathrm{~cm}$ in men and $\geq 88 \mathrm{~cm}$ in women, (2) triglycerides (TG) $\geq 150 \mathrm{mg} / \mathrm{dL}(1.7 \mathrm{mmol} / \mathrm{L})$ or being on drug treatment for elevated triglycerides, (3) high density lipoprotein cholesterol $(\mathrm{HDL}-\mathrm{C})<40 \mathrm{mg} / \mathrm{dL}(1.03 \mathrm{mmol} / \mathrm{L})$ in $\mathrm{men}$ and $<50 \mathrm{mg} / \mathrm{dL}$ $(1.3 \mathrm{mmol} / \mathrm{L})$ in women or being on drug treatment for reduced HDL-C, (4) blood pressure (BP) $\geq 130 \mathrm{mmHg}$ systolic blood pressure (SBP) or $\geq 85 \mathrm{mmHg}$ diastolic blood pressure (DBP) or being on antihypertensive drug treatment, for a patient with a history of hypertension, and (5) fasting plasma glucose (FPG) $\geq 100 \mathrm{mg} / \mathrm{dL}$ or being on drug treatment for elevated glucose [3]. However, the criterion for waist circumference is slightly different for each country and race.

Each metabolic risk factor is associated with one another, and together the risk factors promote atherosclerotic cardiovascular disease [4]. The main underlying risk factors for 
metabolic syndrome are abdominal obesity and insulin resistance $[5,6]$. Therefore, preventing atherosclerotic cardiovascular disease by controlling waist circumference and insulin resistance is the key to managing metabolic syndrome. Clinically, each treatment of hyperglycemia, hypertension, and hyperlipidemia is prescribed according to each patient's state.

However, ACE inhibitor that is a drug used for hypertension, including enalapril and captopril, may cause adverse events such as cough, increased serum creatinine, headache, and skin rash $[7,8]$. It has also been shown that metformin, a drug used to treat type 2 diabetes mellitus, can induce gastrointestinal symptoms and lactic acidosis [6]. Therefore, herbal medicines showing evidence of safety and efficacy can be alternative treatments for metabolic diseases.

Although there are several reviews of herbal medicines for obesity $[9,10]$, hypertension [11], and type 2 diabetes mellitus [12], systematic review for metabolic syndrome has not been conducted yet. This study, however, reviews not only a single disease but also metabolic syndrome as a whole. The aim of this study is to evaluate the efficacy and safety of herbal medicines to help manage metabolic syndrome.

\section{Materials and Methods}

\subsection{Data Source and Search Strategy}

2.1.1. Data Source. This study included the following databases: PubMed, EMBASE, Cochrane Library, SCOPUS, AMED, China National Knowledge Infrastructure (CNKI), KoreaMed, KMBASE, OASIS, Electronic (J-STAGE), and Japan Science and Technology Information Aggregator.

2.1.2. Search Strategy. The study used herbal medicine, metabolic syndrome, and randomized controlled trials for the basic search terms. A search strategy in PubMed is shown in Table 1. Language and publication date were not restricted. The date for the search was December 9, 2015. This review's protocol was registered in PROSPERO (an international prospective register of systematic reviews) (registration number: CRD42014006842).

\subsection{Inclusion Criteria}

2.2.1. Study Design. Randomized controlled trials (RCTs) were included regardless of blinding. Other designs such as in vivo, in vitro, case report, and retrospective study and thesis were excluded.

2.2.2. Participants. Participants were patients with metabolic syndrome and there was no restriction to sex or age. Diagnosis criteria of metabolic syndrome were restricted to international or national standard criteria such as AHA/NHLBI, National Cholesterol Education Program-Adults Treatment Panel (NCEP-ATP), and International Diabetes Federation (IDF) and should be clearly described in Section 2. Chinese pattern identification was optional. Concurrent diseases with metabolic syndrome were excluded.
2.2.3. Interventions. Single or mixed herbal medicines with East Asian medical theory were included. Traditional Chinese Medicine, Traditional Korean Medicine, and Japanese Kampo Medicine are regarded as East Asian medicine. Ayurvedic medicine, crude plant, food, and dietary supplement were excluded. Combination therapy with western medicines, acupuncture, and moxibustion was also excluded. Exercise, diet-control, and health education were not restricted if they were applied to both intervention and control groups.

2.2.4. Comparisons. There was no special restriction on comparisons. Placebo, no treatment, active-control, exercise, diet-control, health education, and usual care were allowed as control groups. Active-control means western medicines for metabolic syndrome, or herbal medicines other than intervention.

2.2.5. Outcome Measures. The primary outcome measures were WC, FPG, TG, HDL-C, SBP, and DBP which are clinical parameters of metabolic syndrome. Secondary outcome measures included body mass index (BMI), body weight, hemoglobin Alc, 2-hour postprandial glucose (2 hPG), total cholesterol (TC), low density lipoprotein cholesterol (LDLC), and clinical effective rate. Trials that assessed one or more outcome measures were included. However, trials which measured level of hormone or enzyme, such as leptin and adiponectin, were excluded in this review.

\subsection{Study Selection and Data Extraction}

2.3.1. Selection of Literature Articles. After excluding any duplication of literature reviews from 10 databases, two authors (S. Jang and J.-S. Park) reviewed titles and abstracts for the first exclusion. Then, full texts of the selected literature articles were subject to another review before the final selection of literature articles was made to make sure each article qualified using the inclusion criteria for this study. For excluded literature articles, the reason for exclusion was recorded. When two authors showed a difference of opinion, a third author (B.-H. Jang) intervened to help come to an agreement. The entire process was displayed by generating a flow diagram in PRISMA (Preferred Reporting Items for Systematic reviews and Meta-Analyses) (Figure 1).

2.3.2. Data Extraction. One author (S. Jang) conducted data extraction, and a different author (Y. Ko) reviewed the data. Items extracted from each trial include the following: (1) general characteristics of the study: author, published year, language, and country; (2) participants: sample size, sex, and age, Chinese pattern identification; (3) interventions: intervention, compositions of intervention, formulation of intervention, control, dosage, and medication period; and (4) outcomes: outcomes, main conclusion, and adverse events.

2.4. Assessment with Risk of Bias. Two authors (S. Jang and Y. Ko) assessed methodological quality using the Risk of Bias (RoB) tool, which was developed by Cochrane [7]. RoB was divided into 6 selection biases, including 2 selection biases 
TABle 1: Search strategy (PubMed).

\begin{tabular}{|c|c|}
\hline \multicolumn{2}{|r|}{ Chinese herbal medicine } \\
\hline$\# 1$ & Chinese medic ${ }^{*}$ \\
\hline$\# 2$ & TCMs or TCM \\
\hline$\# 3$ & Chinese herb* \\
\hline$\# 4$ & Chinese drug* \\
\hline \#5 & Chinese formul ${ }^{*}$ \\
\hline$\# 6$ & Chinese plant* \\
\hline$\# 7$ & Chinese prescri ${ }^{*}$ \\
\hline$\# 8$ & Chinese remed ${ }^{*}$ \\
\hline$\# 9$ & Chinese materia ${ }^{*}$ medica* \\
\hline$\# 10$ & kampo \\
\hline \#11 & herb $^{*}$ medic $^{*}$ \\
\hline$\# 12$ & “Medicine, Chinese traditional” (MeSH Terms) \\
\hline$\# 13$ & "Medicine, East Asian Traditional" (MeSH Terms) \\
\hline$\# 14$ & "Medicine, kampo" (MeSH Terms) \\
\hline$\# 15$ & "Herbal Medicine" (MeSH Terms) \\
\hline \#16 & “drugs, Chinese herbal” (MeSH Terms) \\
\hline$\# 17$ & $\begin{array}{r}\# 1 \text { or } \# 2 \text { or } \# 3 \text { or } \# 4 \text { or } \# 5 \text { or } \# 6 \text { or } \# 7 \text { or } \# 8 \text { or } \# 9 \text { or } \# 10 \text { or } \# 11 \text { or } \# 12 \text { or } \# 13 \text { or } \# 14 \text { or } \\
\# 15 \text { or } \# 16\end{array}$ \\
\hline \multicolumn{2}{|r|}{ Metabolic syndrome } \\
\hline$\# 18$ & “Metabolic Syndrome X” (MeSH Terms) \\
\hline$\# 19$ & metabolic syndrome \\
\hline \#20 & cardiometabolic syndrome \\
\hline \#21 & insulin resistance syndrome \\
\hline \#22 & “syndrome X” \\
\hline$\# 23$ & "Reaven’s syndrome” \\
\hline \#24 & CHAOS AND Australia \\
\hline \#25 & $\# 18$ or $\# 19$ or $\# 20$ or $\# 21$ or $\# 22$ or \#23 or \#24 \\
\hline \multicolumn{2}{|r|}{ Randomized controlled trials } \\
\hline \#26 & $\begin{array}{l}\text { ((clinical (Title/Abstract) AND trial (Title/Abstract)) OR clinical trials (MeSH } \\
\text { Terms) OR clinical trial (Publication Type) OR random* (Title/Abstract) OR } \\
\text { random allocation (MeSH Terms) OR therapeutic use (MeSH Subheadings)) }\end{array}$ \\
\hline \#27 & \#17 AND \#25 AND \#26 \\
\hline
\end{tabular}

(random sequence generation and allocation concealment), performance bias, detection bias, attrition bias, and reporting bias. Each item of all the included RCTs was determined as "high risk," "unclear," or "low risk." A RoB graph was drawn using RevMan 5.3 program.

2.5. Data Analysis. We used mean difference (MD) with 95\% confidential interval (CI) to measure primary outcomes between trials. Analyses were divided into 4 subgroups depending on type of controls: no treatment, placebo, metformin, other western medicines. Heterogeneity was analyzed by the Cochrane $Q$ and $I^{2}$ test. $I^{2}$ values of $25 \%, 50 \%$, and $75 \%$ mean low, medium, and high levels of statistical heterogeneity. RevMan 5.3 program was used for analysis. We also made summary of findings (SoF) table to present results of review by Gradepro software.

\section{Results}

3.1. Description of Included Trials. From ten databases, 1,098 literature articles were identified. Among them, 826 records remained after eliminating duplications, and 733 records were excluded after screening titles and abstracts. By reviewing full texts of 93 records, 12 RCTs were included in this systematic review. The process of the study selection is shown in Figure 1.

3.1.1. Characteristics of Study. Among the 12 included RCTs [13-24], 11 studies [14-24] were written in Chinese and conducted in China, and 1 study [13] was written in English and conducted in India. Four trials $[15,16,19,24]$ selected the IDF guideline for diagnosis of metabolic syndrome, 2 trials $[13,22]$ used the NCEP-ATP guideline, 3 trials $[17,20,23]$ used the Chinese Diabetes Society criteria, and 3 trials used the American Diabetes Association guideline [18], the AACE 


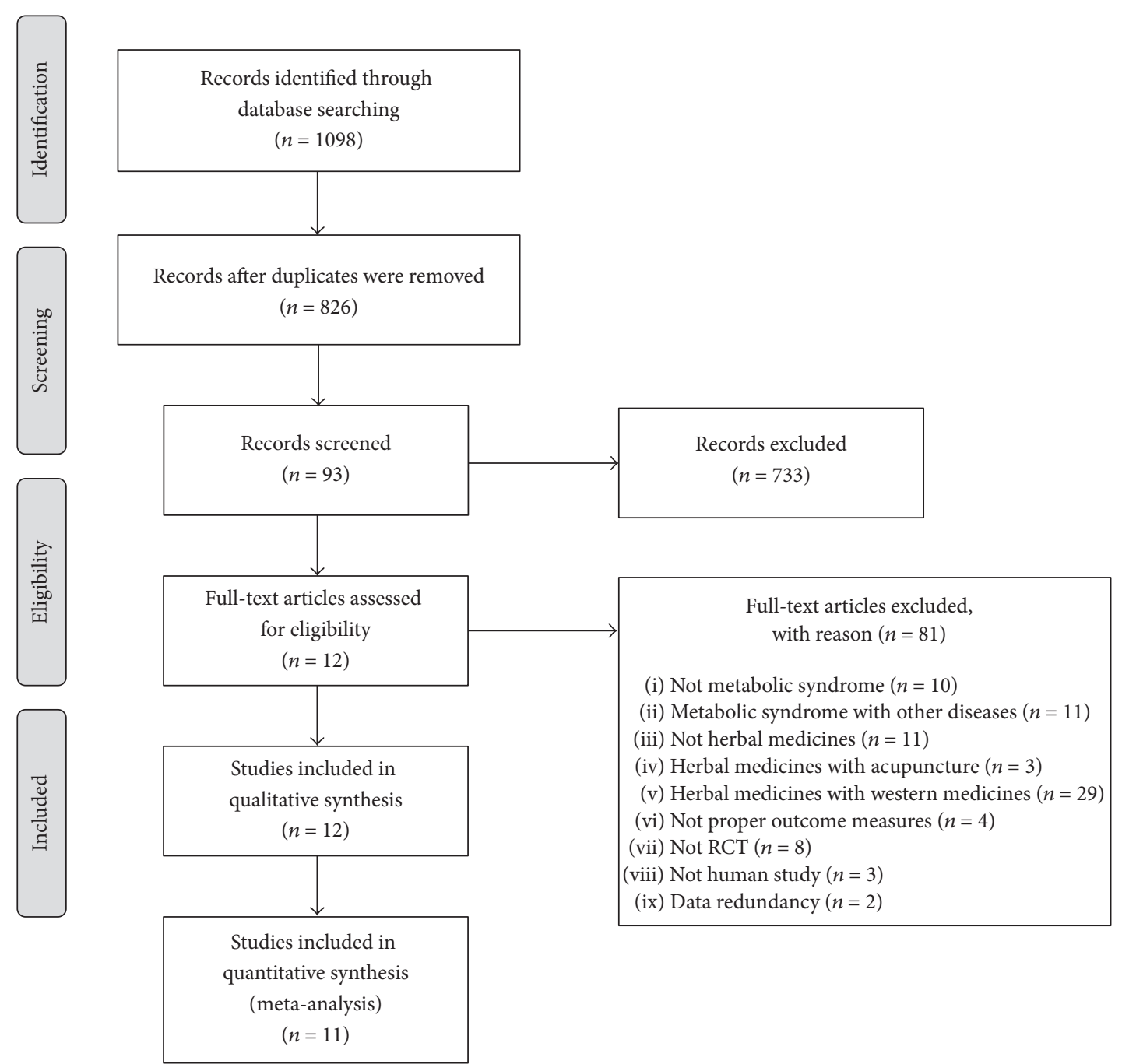

FIgURE 1: The PRISMA flow diagram of study selection.

clinical criteria [21], and the China Dyslipidemia Prevention guideline [14], respectively. Six trials [15, 17-20, 24] additionally applied Chinese pattern identification as the inclusion criterion. There were 4 trials $[15,17,19,20]$ that had a medication period of less than 8 weeks, 3 trials $[18,21,23]$ with a medication period of more than 8 weeks but less than 12 weeks, and 5 trials $[13,14,16,22,24]$ with a medication period of more than 12 weeks. Details are described in Table 2.

3.1.2. Participants. The number of participants for the trials varied from 43 [14] to 183 [13]. No trial was restricted to participants based on sex, but one trial [17] did not report sex distribution. Six trials included Chinese pattern identification as inclusion criteria: 2 trials $[15,19]$ of Exuberance of PhlegmDampness Type, 1 trial [24] of Spleen Deficiency and Stagnation of Dampness Type, 1 trial [18] of Heart-Liver Stagnated Heat Type, 1 trial [20] of Flaming-Up of Fire of the Liver Type, and 1 trial [17] of Blood-Stasis Type. Two trials [14, 20] were conducted on patients with hypertension and metabolic syndrome.
3.1.3. Comparisons. Comparisons were divided into two types. One type was a placebo or no treatment, and 2 trials [13, 14] were compared with a placebo, while 3 trials [15-17] were conducted under no treatment. Diet-control and exercise or health education was used for the no treatment group. The other type was western medicine, and 7 trials [18-24] followed this comparison type. Metformin was used as a comparison in 3 trials $[18,19,21]$, and nifedipine was used in 1 trial [20]. Two trials [22-24] provided different conventional medicines according to the symptoms of the patients.

3.2. Effects of Interventions. Overall efficacy of FPG, TG, SBP, DBP, WC, and HDL-C was presented in summary of findings (Table 3). Meta-analyses of FPG, TG, SBP, and DBP were shown in Figures 2-5. All 12 trials had different herbal medicine interventions; therefore, we also compared effects of each intervention. Table 4 shows the mean differences (MD) for each outcome measure. The unit $\mathrm{mmol} / \mathrm{L}$ was converted into $\mathrm{mg} / \mathrm{dL}$. 


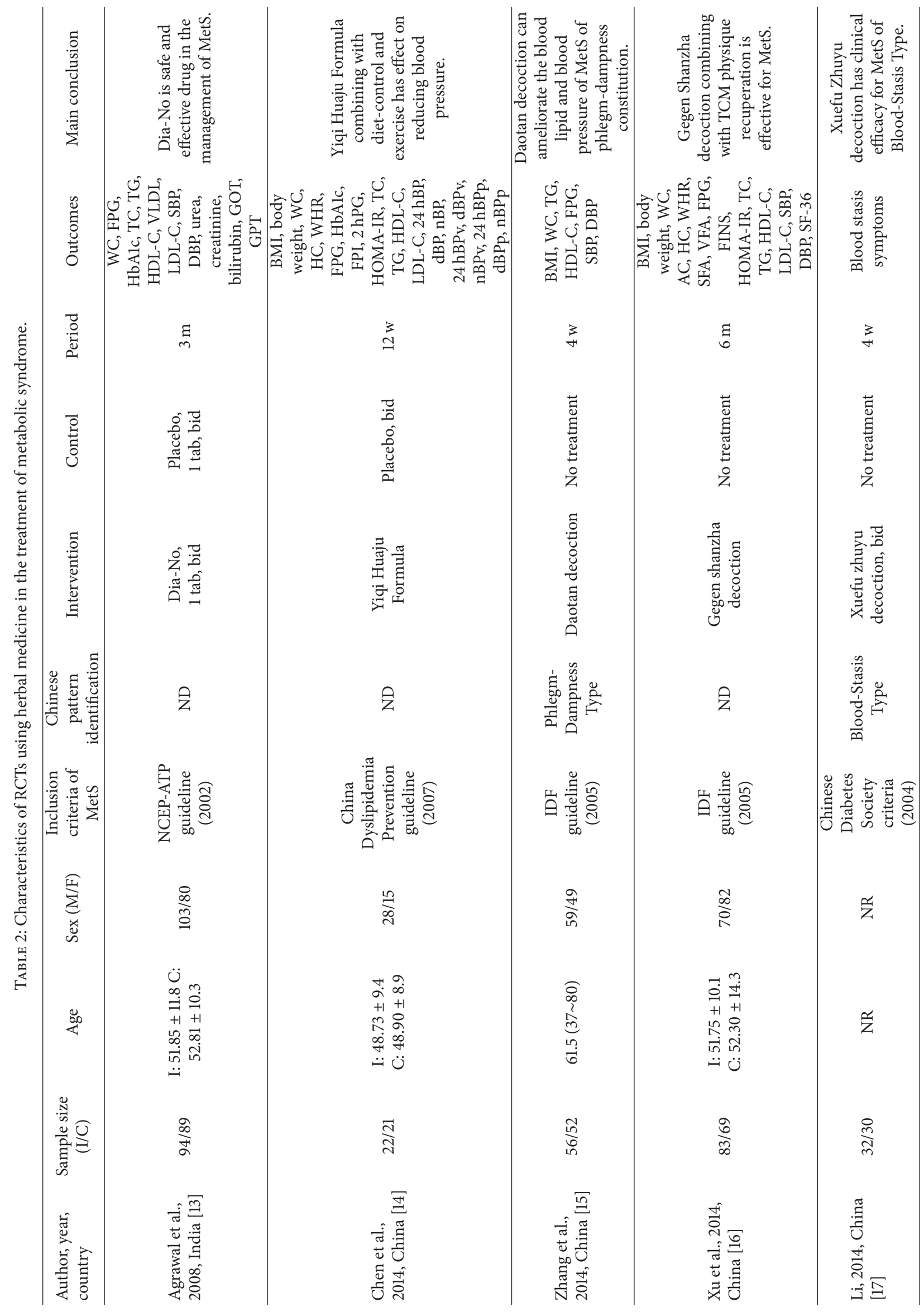




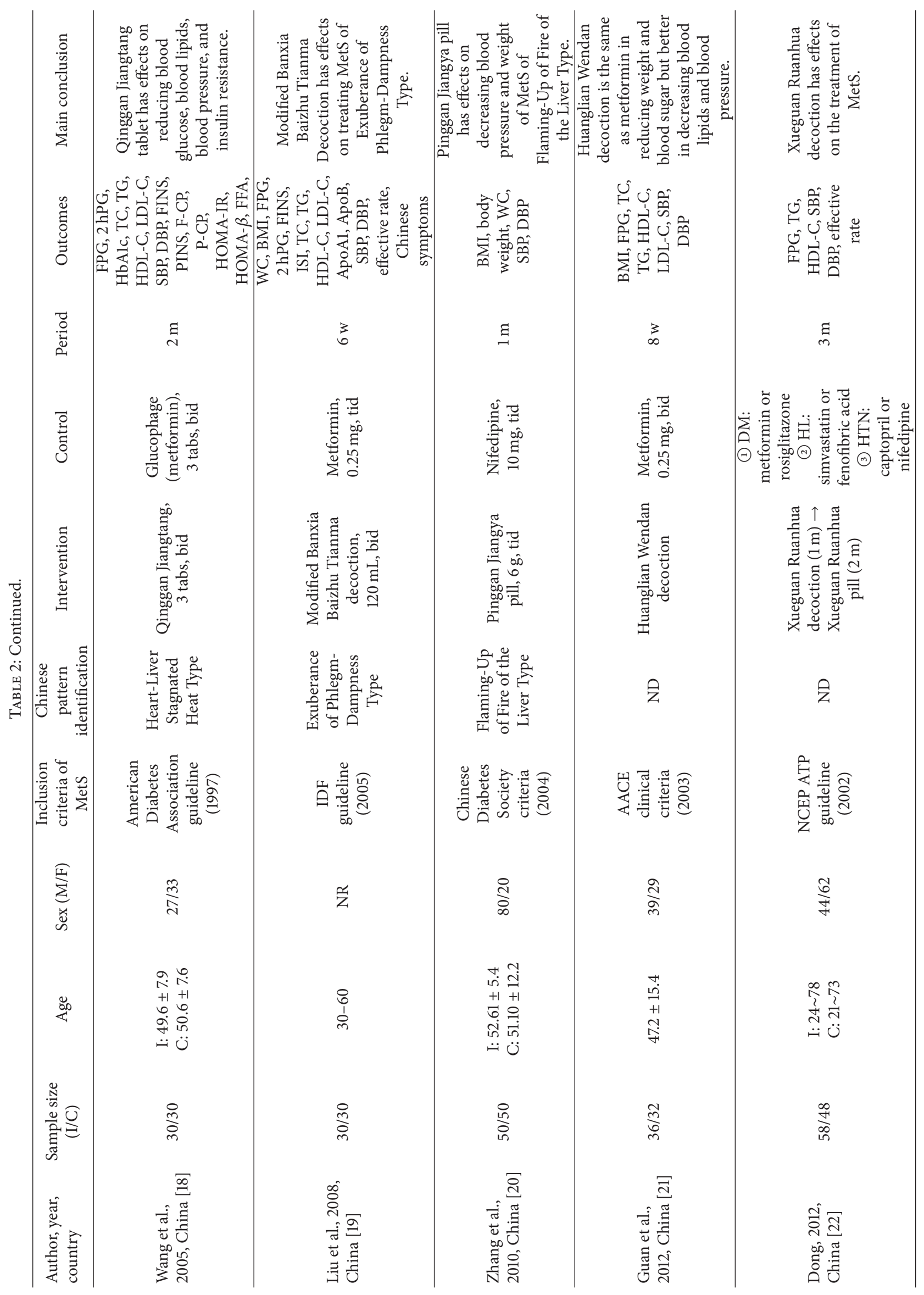




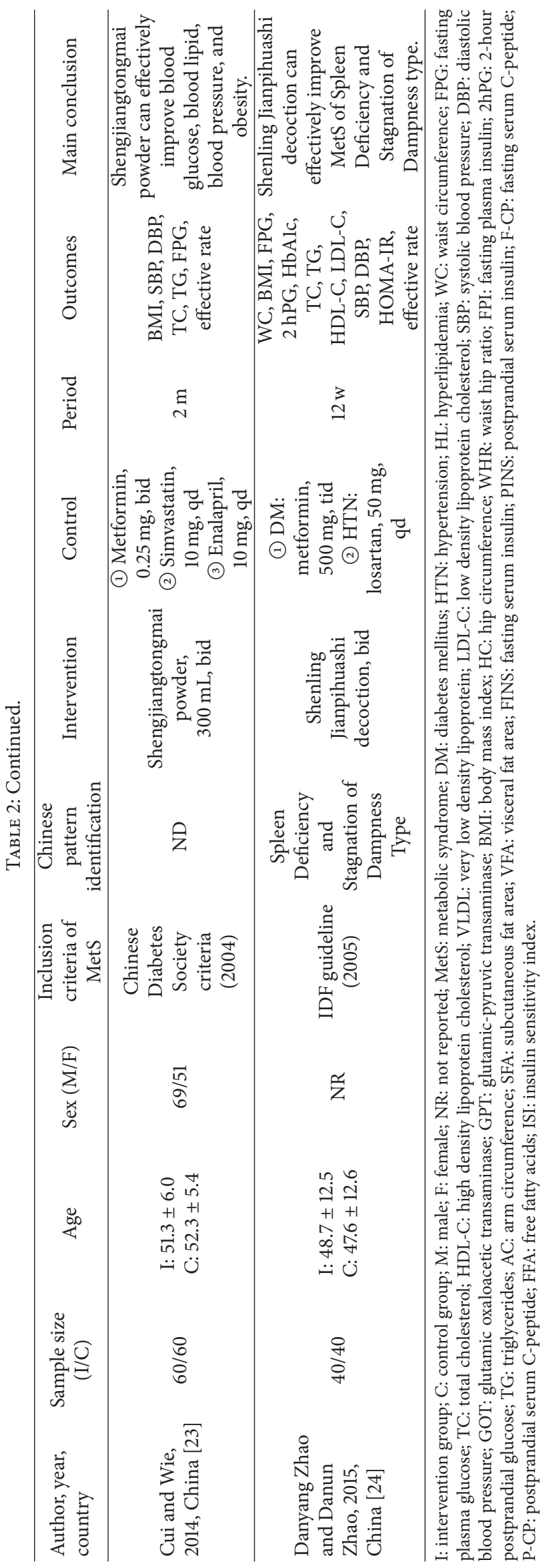




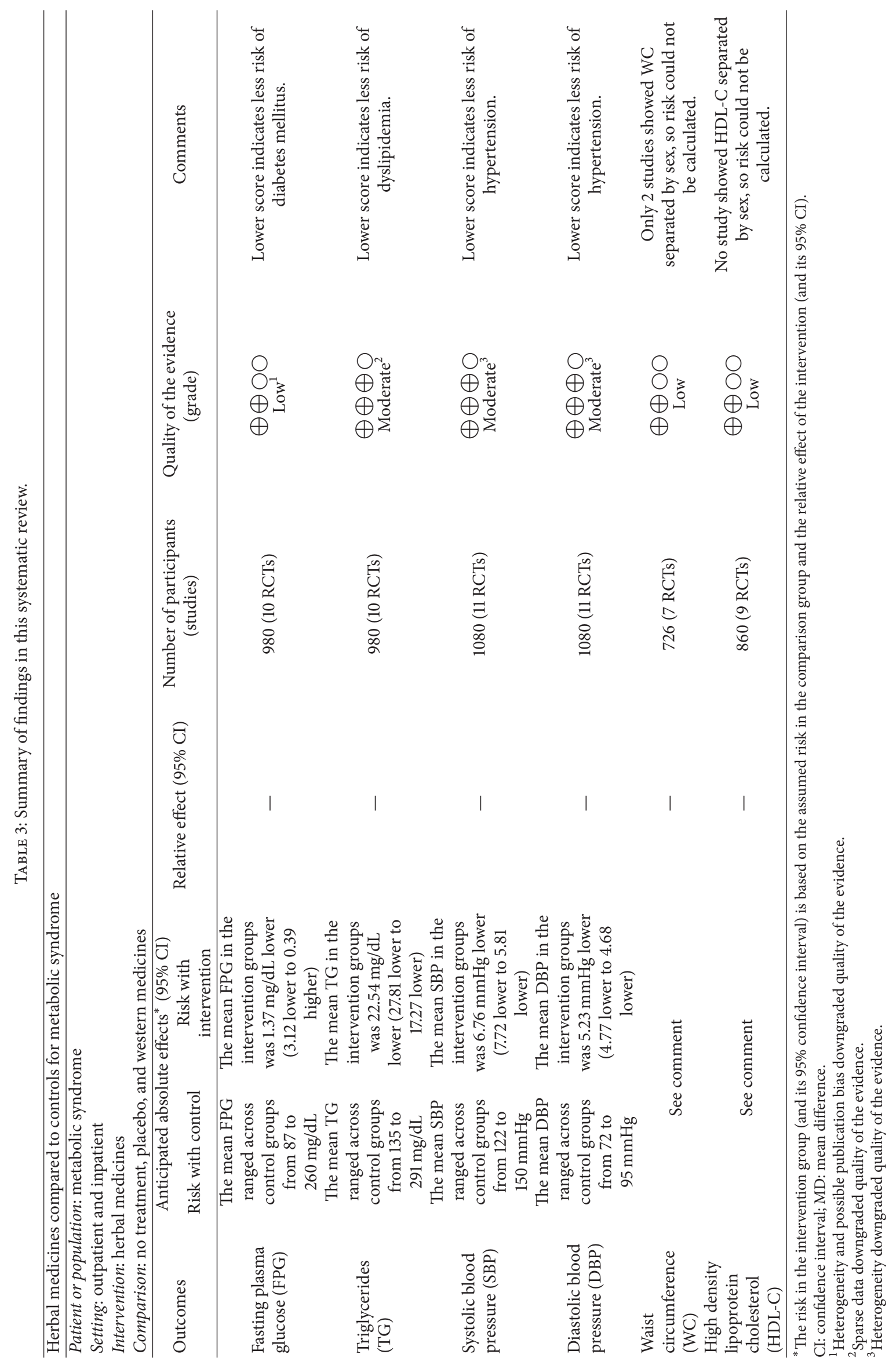


TABLE 4: Estimate effects of herbal medicines for metabolic syndrome: differences of values before and after treatment.

\begin{tabular}{|c|c|c|c|c|c|c|c|c|c|}
\hline Intervention & Study ID & WC & BMI & FPG & $2 \mathrm{hPG}$ & TG & HDL-C & SBP & DBP \\
\hline Dia-No & $\begin{array}{l}\text { Agrawal et al., } \\
2008 \text { [13] }\end{array}$ & +0.02 & $-0.18^{*}$ & $-41.15^{*}$ & NR & $-10.59^{*}$ & +1.12 & $-2.29^{*}$ & $-1.23^{*}$ \\
\hline Yiqi Huaju Recipe & $\begin{array}{c}\text { Chen et al., } 2014 \\
{[14]}\end{array}$ & $-4.68^{*}$ & $-1.51^{*}$ & $-7.02^{\#}$ & $-29.34^{*}$ & -7.12 & +1.93 & $-11.32^{*}$ & $-6.5^{*}$ \\
\hline Daotan decoction & $\begin{array}{l}\text { Zhang et al., } \\
2014 \text { [15] }\end{array}$ & $-2.98^{*}$ & $-0.49^{*}$ & -2.52 & NR & $-28.48^{*}$ & $+2.32^{*}$ & $-7.43^{*}$ & $-2.28^{*}$ \\
\hline Gegen Shanzha decoction & $\begin{array}{c}\text { Xu et al., } 2014 \\
{[16]}\end{array}$ & $-5.31^{*}$ & $-2.69^{*}$ & $-21.42^{*}$ & NR & $-99.68^{*}$ & $+18.91^{*}$ & $-19.66^{*}$ & $-10.5^{*}$ \\
\hline Xuefu Zhuyu decoction & $\mathrm{Li}, 2014[17]$ & NR & NR & NR & NR & NR & NR & NR & NR \\
\hline Qinggan Jiangtang tablet & $\begin{array}{c}\text { Wang et al., } \\
2005 \text { [18] }\end{array}$ & NR & NR & $-28.8^{\#}$ & $-52.2^{\#}$ & $-12.46^{*}$ & +2.70 & $-7.5^{*}$ & $-2.9^{*}$ \\
\hline Modified Banxia Baizhu Tianma decoction & $\begin{array}{c}\text { Liu et al., } 2008 \\
{[19]}\end{array}$ & -0.91 & $-0.74^{\#}$ & $-7.02^{\#}$ & $-19.08^{\#}$ & $-23.14^{*}$ & $+7.72^{*}$ & $-4.38^{*}$ & $-3.23^{*}$ \\
\hline Pinggan Jiangya pill & $\begin{array}{l}\text { Zhang et al., } \\
2010 \text { [20] }\end{array}$ & $-7.81^{*}$ & $-2.95^{*}$ & NR & NR & NR & NR & $-28.00^{*}$ & $-14.33^{*}$ \\
\hline Huanglian Wendan decoction & $\begin{array}{c}\text { Guan et al., } 2012 \\
\text { [21] }\end{array}$ & NR & $-1.95^{\#}$ & $-27.54^{\#}$ & NR & $-62.3^{*}$ & $+11.19^{*}$ & $-10.73^{*}$ & $-8.24^{*}$ \\
\hline Xueguan Ruanhua decoction & Dong, 2012 [22] & NR & NR & -6.12 & NR & $-30.26^{*}$ & $+15.83^{*}$ & $-8.75^{*}$ & $-9.33^{*}$ \\
\hline Shengjiangtongmai powder & $\begin{array}{l}\text { Cui and Wie, } \\
2014[23]\end{array}$ & NR & -2.52 & $-82.08^{*}$ & NR & $-89.89^{*}$ & NR & $30^{*}$ & $15^{*}$ \\
\hline Shenling Jianpihuashi decoction & $\begin{array}{l}\text { Danyang Zhao } \\
\text { and Danun } \\
\text { Zhao, } 2015[24]\end{array}$ & $-7.2^{*}$ & $-2.7^{\#}$ & $-16.2^{\#}$ & $-34.2^{\#}$ & $-71.2^{*}$ & +3.86 & $-11.6^{\#}$ & $-9.3^{\#}$ \\
\hline
\end{tabular}

* Significant difference between intervention and control group $(P<0.05)$.

"Significant difference before and after treatment $(P<0.05)$.

WC: waist circumference; BMI: body mass index; FPG: fasting plasma glucose; 2 hPG: 2-hour postprandial glucose; TG: triglycerides; HDL-C: high density lipoprotein cholesterol; SBP: systolic blood pressure; DBP: diastolic blood pressure.

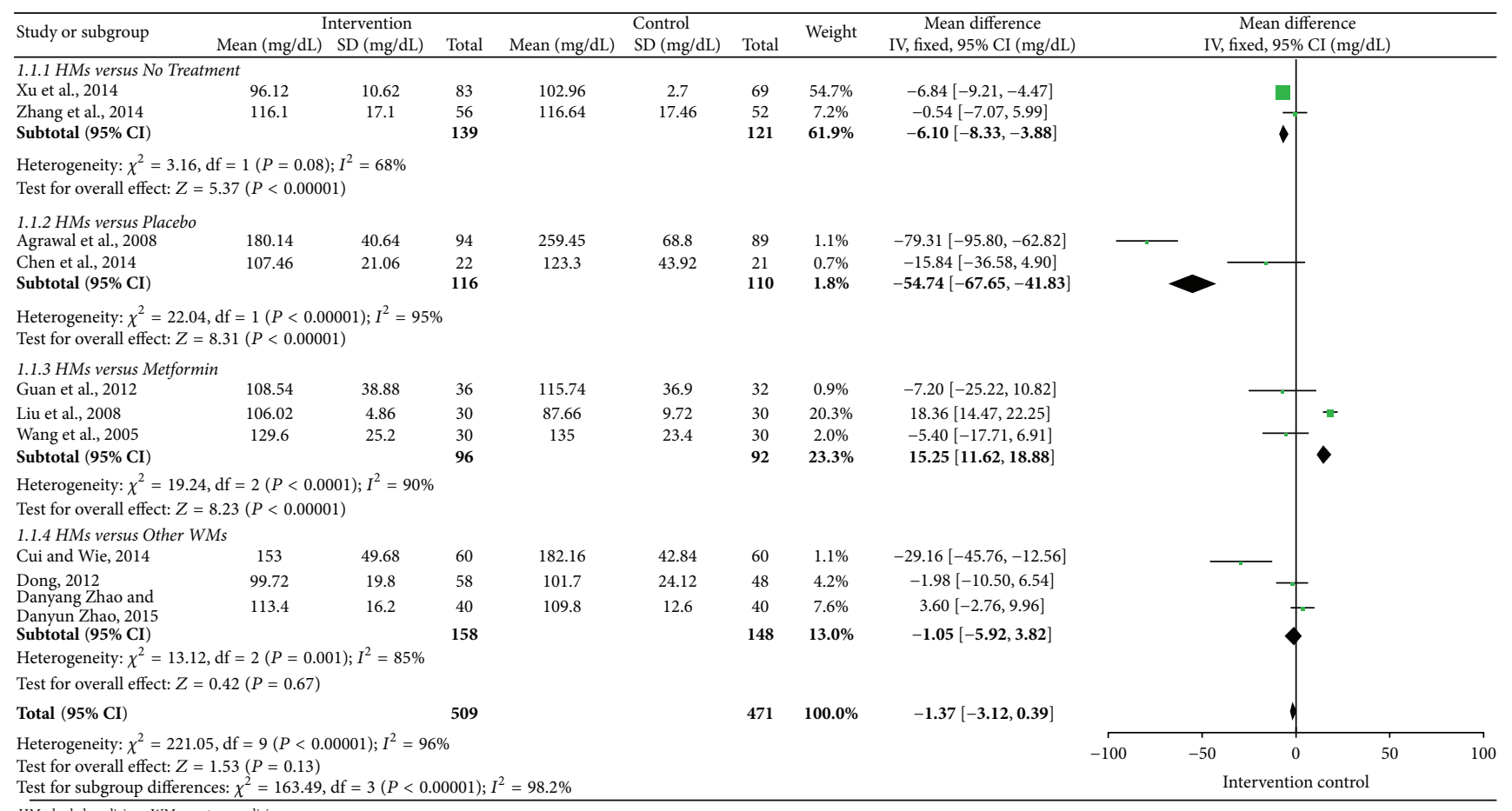

FIgURE 2: Forest plot for fasting plasma glucose (FPG). 


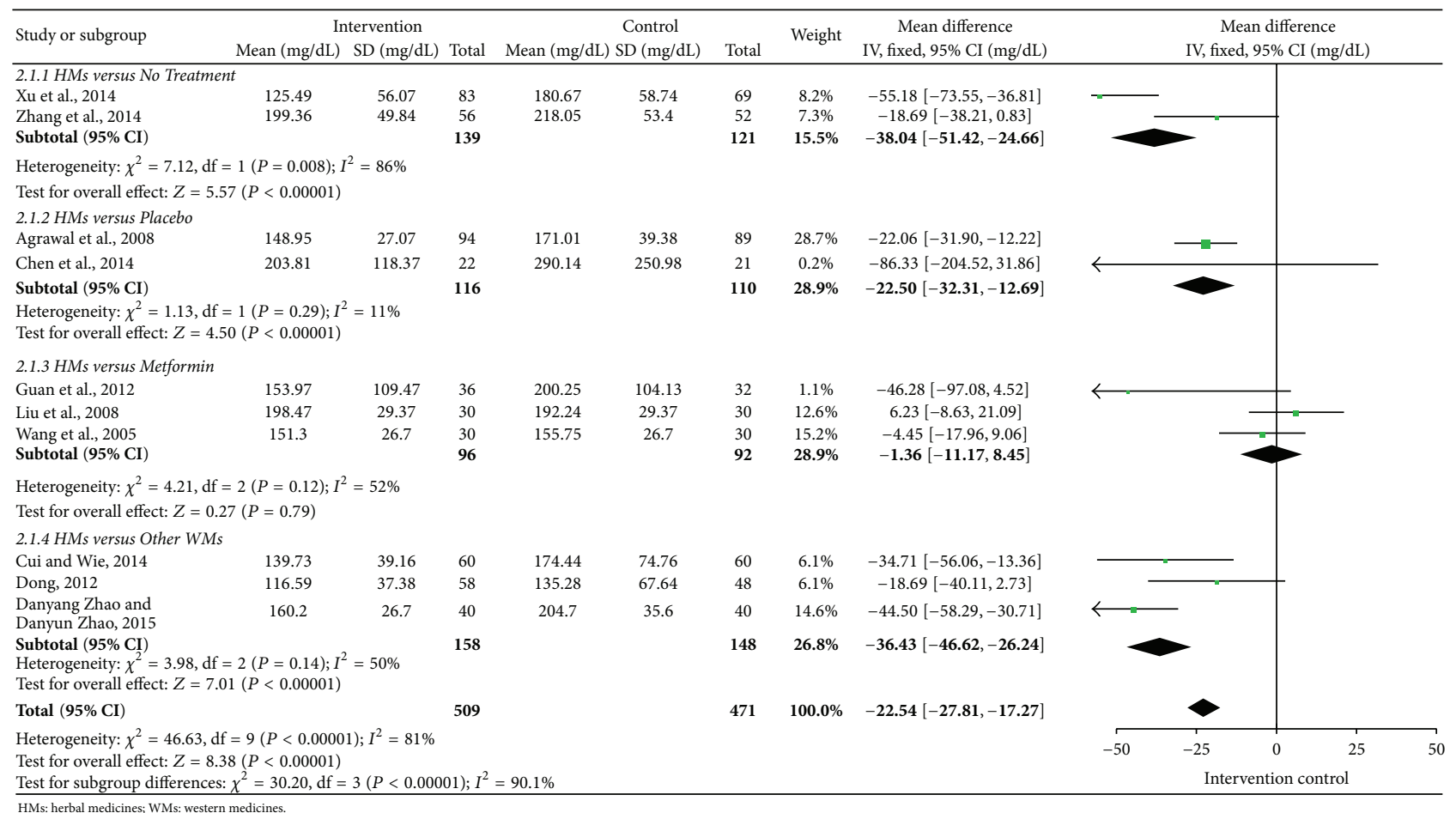

FIgURE 3: Forest plot for triglycerides (TG).

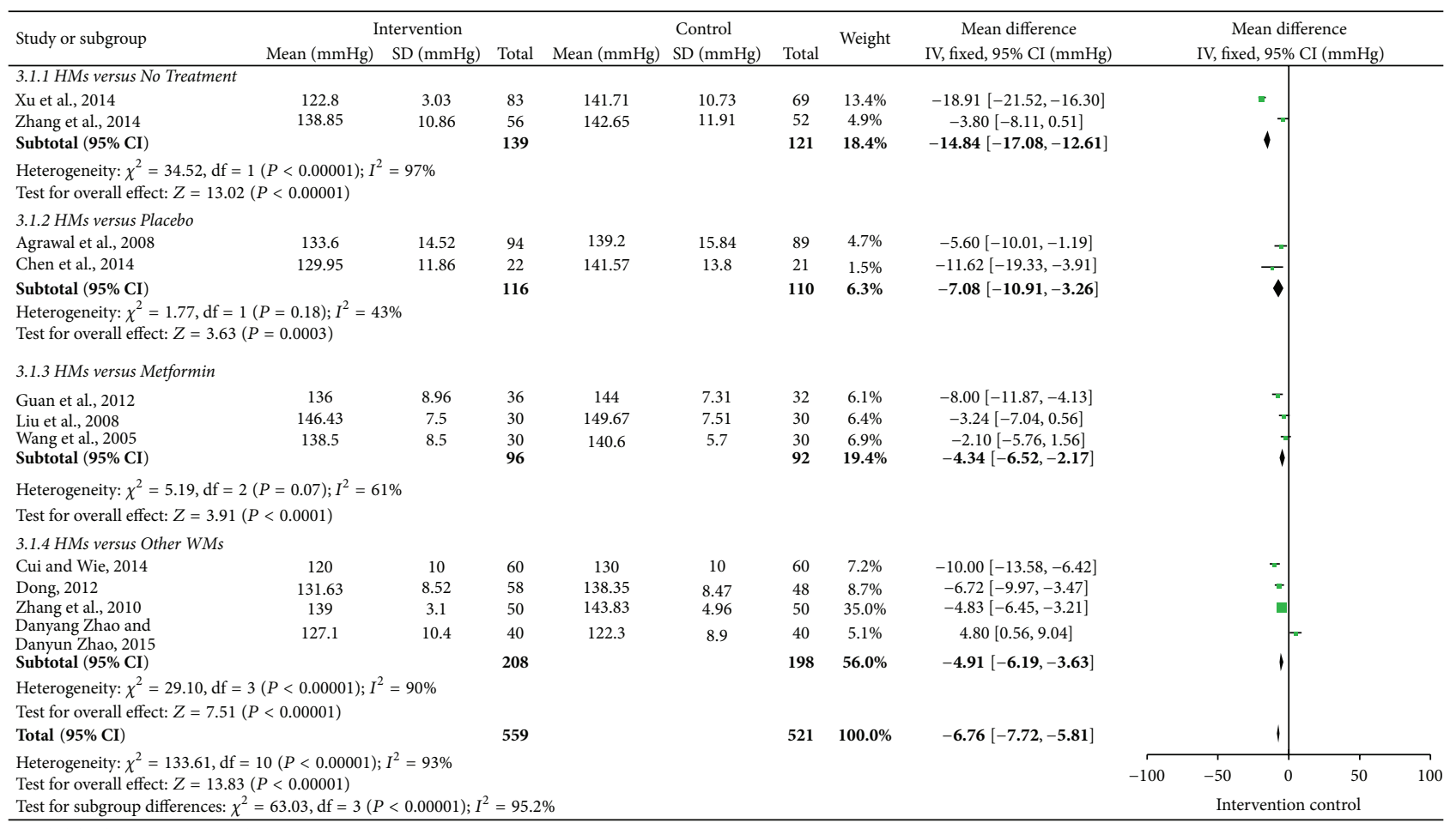

HMs: herbal medicines: WMs: western medicines.

FIGURE 4: Forest plot for systolic blood pressure (SBP). 


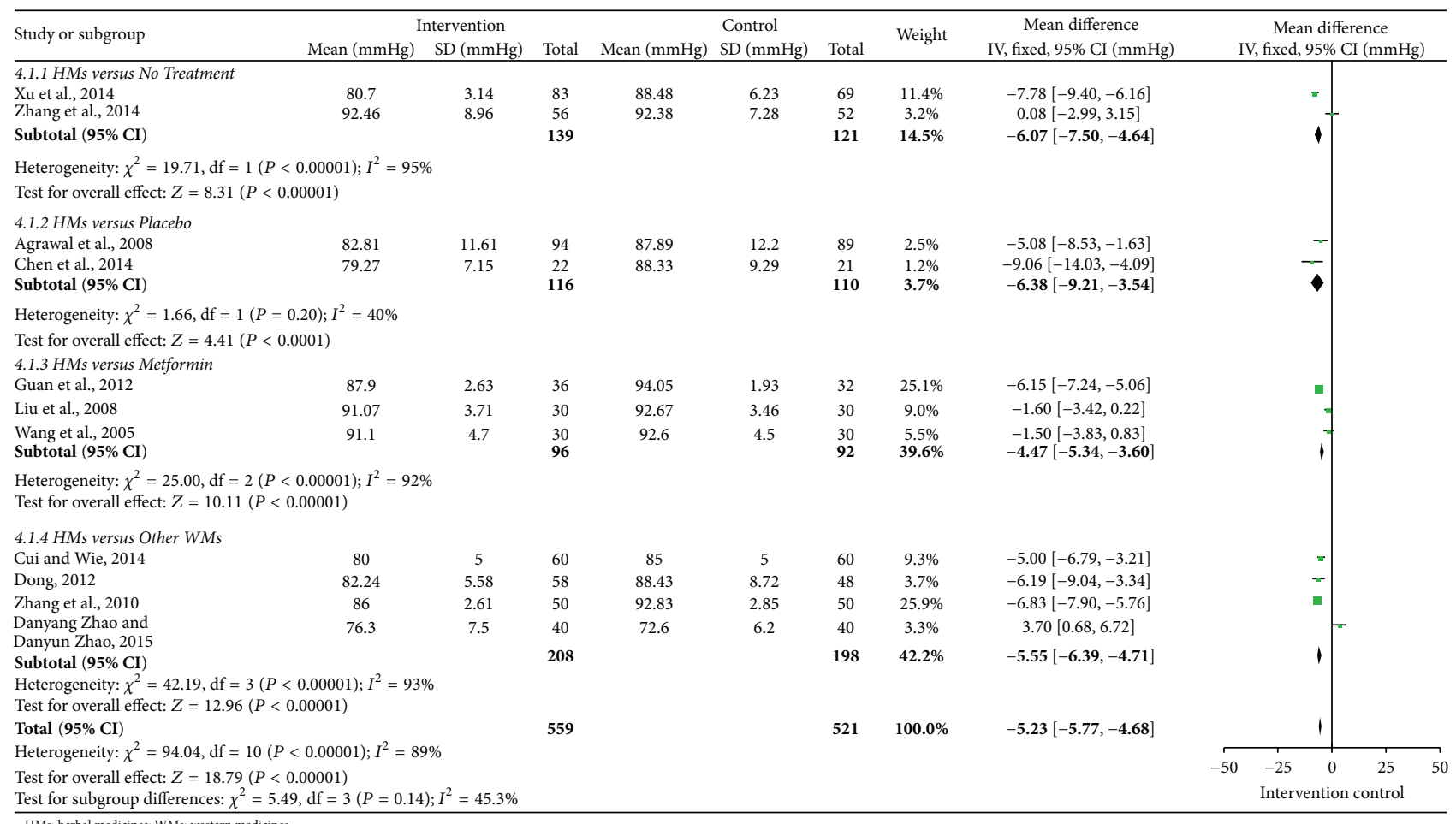

FIGURE 5: Forest plot for diastolic blood pressure (DBP).

3.2.1. Waist Circumference and Body Mass Index. There were seven trials $[13-16,19,20,24]$ reporting WC. Five trials [14$16,20,24]$ showed significant reductions of WC compared with the control groups: Yiqi Huaju Recipe (MD: $-4.68, n=$ 43), Daotan decoction (MD: $-2.98, n=108$ ), Gegen Shanzha decoction (MD: $-5.31, n=152$ ), Pinggan Jianya pill (MD: -7.81, $n=100$ ), and Shenling Jianpihuashi decoction (MD: $-7.2, n=80$ ). Modified Banxia Baizhu Tianma decoction [19] showed less effect on decreasing WC than metformin. There was a slight increase in one trial [13]; however, it was not statistically significant.

There were nine trials [13-16, 19-21, 23, 24] reporting BMI. Six trials [13-16, 20, 23] showed significant reductions of BMI compared with the control groups: Dia-No decoction (MD: $-0.18, n=183$ ), Yiqi Huaju Recipe (MD: $-1.51, n=43$ ), Daotan decoction (MD: $-0.49, n=108$ ), Gegen Shanzha decoction (MD: $-2.69, n=152$ ), Pinggan Jiangya pill (MD: $-0.74, n=100$ ), and Shengjiangtongmai powder (MD: -2.52 , $n=120)$. There were significant reductions after treatment in the remaining 3 trials $[19,21,24]$; however, it was not significant when compared with the control groups. These trials included the Modified Banxia Baizhu Tianma decoction (MD: $-0.74, n=60$ ), Huanglian Wendan decoction (MD: $-1.95, n=68$ ), and Shenling Jianpihuashi decoction (MD: $-2.7, n=80)$.

3.2.2. Blood Glucose. There were 10 trials $[13-16,18,19,21-$ $24]$ reporting FPG and 4 trials $[14,18,19,24]$ reporting $2 \mathrm{hPG}$. Three trials $[13,16,23]$ showed significant reductions of FPG compared with the control groups: Dia-No decoction (MD: -41.15, $n=183$ ), Gegen Shanzha decoction (MD: $-21.42, n=$ 152 ), and Shengjiangtongmai powder (MD: $-82.08, n=120$ ). Five trials had an effect on lowering FPG; however, effects of herbal medicines were not more than the controls: Yiqi Huaju Recipe (MD: $-7.02, n=43$ ), Qinggan Jiangtang tablet (MD: -28.8, $n=60$ ), Modified Banxia Baizhu Tianma decoction (MD: $-7.02, n=60$ ), Huanglian Wendan decoction (MD: $-27.54, n=68$ ), and Shenling Jianpihuashi decoction (MD: $-16.2, n=80$ ). Daotan decoction [15] and Xueguan Ruanhua decoction [22] were not effective in decreasing FPG. As shown in Figure 2, mean FPG in the intervention groups was 1.37 lower than control groups within 10 trials ( -3.13 to 0.39 ).

For $2 \mathrm{hPG}$, four trials $[14,18,19,24]$ showed significant reductions after treatment, and 1 trial [14] demonstrated an effect when compared with the control group: Yiqi Huaju Recipe (MD: $-29.34, n=43$ ).

3.2.3. Blood Lipids. There were 10 trials [13-16, 18, 19, 21-24] reporting TG and 9 trials $[13-16,18,19,21,22,24]$ reporting HDL-C. Nine trials $[13,15,16,18,19,21-24]$ showed significant reductions of TG compared with the control groups: Dia-No decoction (MD: $-10.59, n=183$ ), Daotan decoction (MD: -28.48, $n=108$ ), Gegen Shanzha decoction (MD: -99.68, $n=152$ ), Qinggan Jiangtang tablet (MD: $-12.46, n=60$ ), Modified Banxia Baizhu Tianma decoction (MD: $-23.14, n=$ $60)$, Huanglian Wendan decoction (MD: $-62.3, n=68$ ), Xueguan Ruanhua decoction (MD: $-30.26, n=106)$, Shengjiangtongmai powder (MD: $-71.2, n=120$ ), and Shenling Jianpihuashi decoction (MD: $-89.89, n=80$ ). As shown 
in Figure 3, mean TG in the intervention groups was 22.54 lower than control groups within 10 trials $(-27.81$ to -17.27$)$.

Five trials $[15,16,19,21,22]$ demonstrated significant increases of HDL-C: Daotan decoction (MD: $+2.32, n=108)$, Gegen Shanzha decoction (MD: $+18.91, n=152$ ), Modified Banxia Baizhu Tianma decoction (MD: $+7.72, n=60)$, Huanglian Wendan decoction (MD: $+11.19, n=68$ ), and Xueguan Ruanhua decoction (MD: $+15.83, n=106)$. Yiqi Huaju Recipe [14] was not effective in improving either TG or HDL-C.

3.2.4. Blood Pressure. There were 11 trials [13-16, 18-24] reporting systolic and diastolic blood pressure. All 11 trials showed significant decreases in blood pressure: DiaNo decoction (MD: $-2.29 /-1.23, n=183$ ), Yiqi Huaju Recipe (MD: $-11.32 /-6.5, n=43$ ), Daotan decoction (MD: $-7.43 /-2.28, n=108$ ), Gegen Shanzha decoction (MD: $-19.66 /-10.5, n=152$ ), Qinggan Jiangtang tablet (MD: $-7.5 /-2.9, n=60)$, Modified Banxia Baizhu Tianma decoction (MD: $-4.38 /-3.23, n=60$ ), Pinggan Jiangya pill (MD: $-28.00 /-14.33, n=100$ ), Huanglian Wendan decoction (MD: $-10.73 /-8.24, n=68$ ), Xueguan Ruanhua decoction (MD: $-8.75 /-9.33, n=106)$, Shengjiangtongmai powder (MD: $-30 /-15, n=120)$, and Shenling Jianpihuashi decoction (MD: $-11.6 /-9.3, n=80$ ). Ten herbal medicines (except Shenling Jianpihuashi decoction [24]) had more effect than the controls, and Shenling Jianpihuashi decoction was not inferior to nifedipine.

The mean SBP was 6.76 lower in the intervention groups compared to control groups within 11 trials $(-7.72$ to -5.81$)$ (Figure 4). The mean DBP was 5.23 lower in the intervention groups than control groups within 11 trials $(-5.77$ to -4.86$)$ (Figure 5).

3.3. Adverse Events and Safety. Six RCTs [13, 14, 20-22, 24] reported 26 adverse events. Nine cases occurred in the herbal medicine group, and the remaining 17 cases occurred in the western medicine group. There was no adverse event in the placebo or no treatment control group. The Dia-No group [13] had 6 upper digestive disorders, the Huanglian Wendan decoction group [21] had 1 gastrointestinal disorder, and the Yiqi Huaju Recipe group [14] had 2 skin hypersensitivities. There was no observed adverse event in the Pinggan Jiangya pill group [20], the Xueguan Ruanhua decoction group [22], and the Shenling Jianpihuashi decoction group [24]. The most commonly reported symptoms were digestive disorders such as nausea, vomiting, and burning of the epigastrium (Table 5).

3.4. Assessment with Risk of Bias. RoB of the 12 RCTs was assessed into 6 areas. Six RCTs [13-18] used a random number table to generate the random sequence. There were high risks of performance bias (blinding of participants and personnel) in 10 trials [15-24] due to the difference of drug formulation. Except in 2 RCTs $[17,20]$ where the primary outcomes were blood pressure and blood-stasis symptom, the remaining 10 trials were assessed as "low risk" for detection bias (blinding of outcome assessment). There were low risks of attrition bias (incomplete outcome data) and reporting bias (selective reporting) in all 12 trials. Only 1 RCT [13] was assessed as "low risk" for all six items. Details of RoB are presented in Figure 6.

\section{Discussion}

As a result of searching 10 databases, 12 randomized controlled trials were included in the systematic review. Because the review's purpose was to determine the efficacy and safety of herbal medicines for metabolic syndrome, clinical trials that included herbal medicines combined with conventional western medicines were excluded. Five trials [13-17] studied herbal medicines versus a placebo or no treatment, and seven trials [18-24] studied herbal medicines versus western medicines. All 12 trials included controls for diet-control, exercise, or health education with medications.

According to Table 4, all the trials showed positive effects with the administration of herbal medicines, and most of them proved significant. Gegen Shanzha decoction [16] improved 5 metabolic indexes, including WC, FPG, TG, HDL-C, and BP. Yiqi Huaju Recipe [14] had an effect on lowering body weight, blood sugar, and blood pressure (except blood lipids). Because the outcome measured the blood-stasis symptom only in the trial for Xuefu Zhuyu decoction [17], the efficacy of Xuefu Zhuyu decoction on metabolic diseases could not be determined. The Pinggan Jiangya pill [20] was effective for the metabolic syndrome of Flaming-Up of Fire of the Liver Type. Flaming-Up of Fire of the Liver Type is the largest type of hypertension [25]; therefore, the Pinggan Jiangya pill would be suitable to treat obesity and hypertension. The Qinggan Jiangtang tablet [18], Modified Banxia Baizhu Tianma decoction [19], Huanglian Wendan decoction [21], Xueguan Ruanhua decoction [22], the Shengjiangtongmai powder [23], and Shenling Jianpihuashi decoction [24] were superior (or not inferior) to western medicines used to treat metabolic syndrome.

As for summary of findings (Table 3), mean differences of metabolic parameters were compared. Metabolic syndrome is not determined by single indicator, and comparing value of each parameter is not appropriate, strictly speaking. Relative risk (RR) of metabolic syndrome should be calculated. However, there was no study presenting difference of prevalence before and after treatment. Meanwhile, WC and HDL-C could not be calculated because they were not separated by men and women within included trials.

Forest plots of FPG, TG, SBP, and DBP indicate high heterogeneity although subgroup analysis was done (Figures $2-5)$. It is assumed that heterogeneity did not result from controls. Instead, difference of each intervention would have been affected. It is also limitation of meta-analysis in this review.

Regarding safety, adverse events were reported less in herbal medicines than in western medicines (Table 5). In Zhang et al's trial [20], the nifedipine group had 4 facial flushes, while the Pinggan Jiangya group had no adverse events. Additionally, there was 1 gastrointestinal disorder with Huanglian Wendan decoction, but there were 5 adverse events when metformin was used in Guan et al.s trial [21]. Therefore, herbal medicines would be an effective and safe 


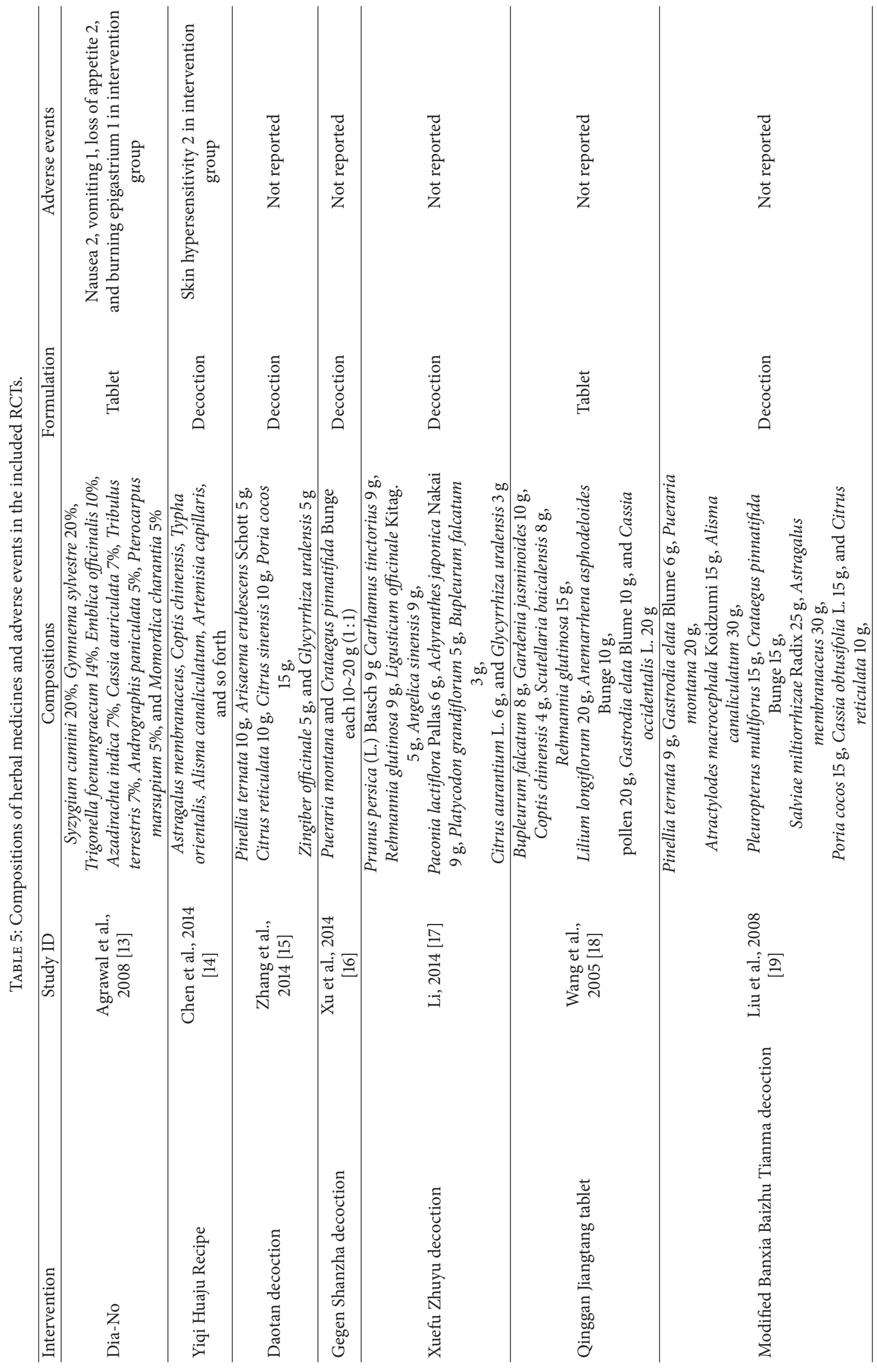




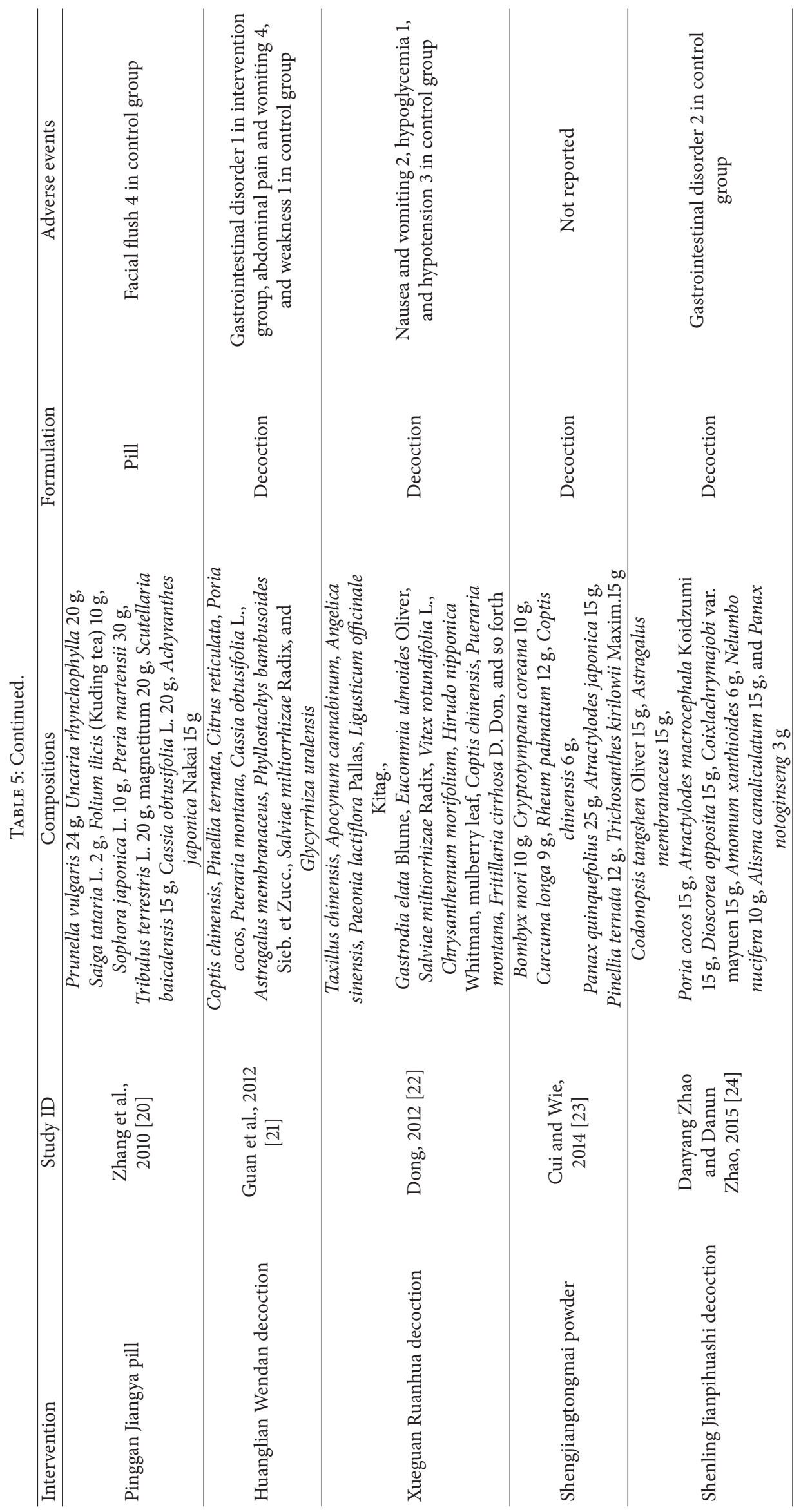




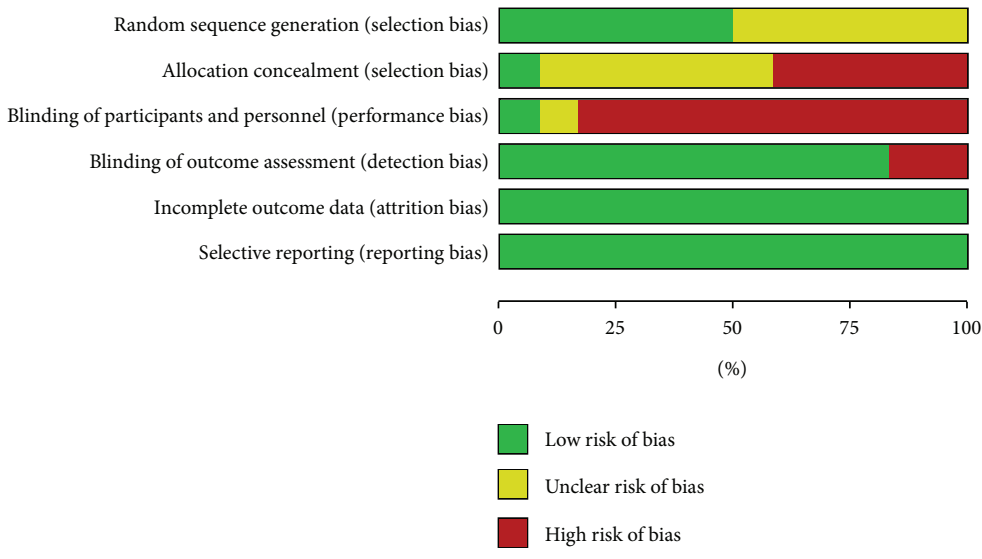

(a)

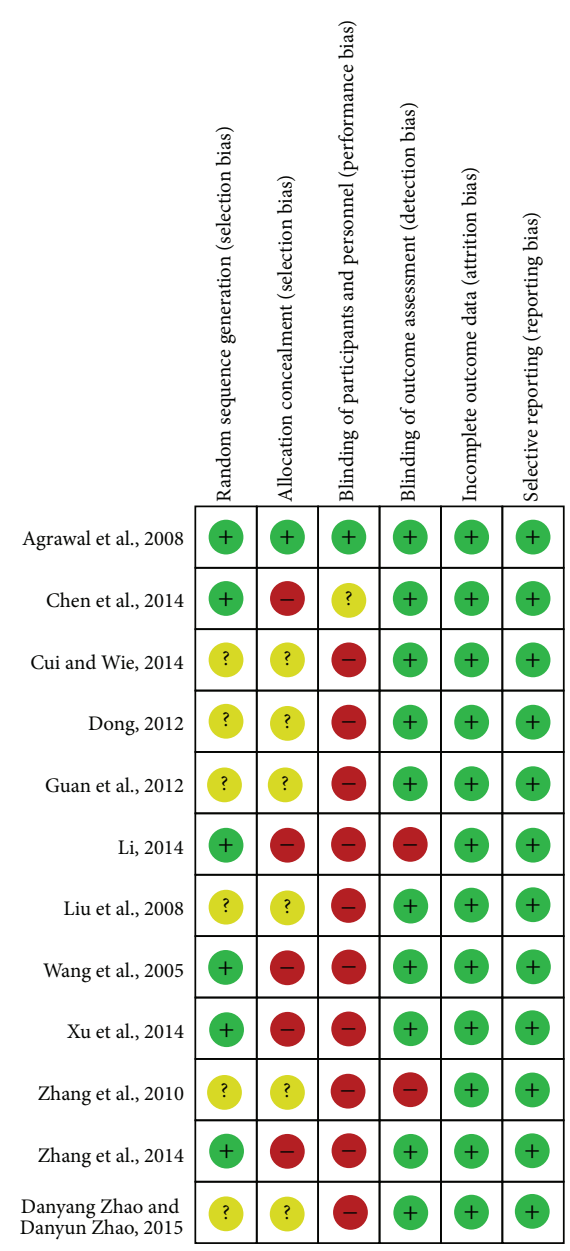

(b)

FiguRE 6: (a) Risk of bias graph: reviewers' assessments about each risk of bias item presented as percentages of all included studies. (b) Risk of bias summary: assessments about each risk of bias item for each included study. "+”: low risk, "?”: unclear risk, and “-”: high risk.

treatment for metabolic syndrome compared with western medicines.

In 6 trials $[15,17-20,24]$, oriental pattern identification along with metabolic factors was set for inclusion criteria. A selection of treatments with pattern identifications would help reduce metabolic risk factors, improve general conditions, and decrease chances of adverse events. With the collection of such trial data, this would provide a ground for herbal medicines to be used as treatment for obesity or metabolic syndrome by pattern identifications.

There are more studies on herbal medicines for metabolic syndrome although they were not included in this review. Keishibukuryogan [26], Yiqi Huaju Qingli Formula [27], BaWei-Wan [28], Heqi San [29], Baoling decoction [30], and Combination of Four Gentlemen Decoction and Sini Powder [31] showed effects on metabolic syndrome; however, trials on these were excluded because treatment group was also treated with conventional western medicines. Herbal supplements, for example, Ginseng [32], berberine, bitter melon [33], nigella sativa [34], and Gymnema sylvestre [35], are also used for management of metabolic diseases. In particular it is well known that ginsenosides which are compounds of ginseng have clear effect of regulating blood glucose and blood pressure [33].

This review has some limitations. First, metabolic syndrome is not a single disorder but rather a complex disease. The herbal medicines used as interventions and their efficacy need to be matched for the following indicators: waist circumference, body weight, blood glucose, blood lipids, and blood pressure. Second, the trials included in the study showed a relatively low level of quality because most of them failed to conduct a double-blinded technique, and only 1 trial [13] met the qualification for advanced protocol. Because of these limitations, there may be the possibility that therapeutic effects have been overestimated. Publication bias also needs to be taken into consideration. Third, there could be trials missing even though we tried to cover all of the RCTs from English, Korean, Chinese, and Japanese databases. However, the study is significant because it has reviewed RCTs on the administration of herbal medicines for treating metabolic 
diseases. Further studies are needed to develop new herbal medicines for metabolic syndrome and to build evidence on their effectiveness and safety.

\section{Conclusion}

Herbal medicines showed therapeutic effects on regulating waist circumference, blood glucose, blood lipids, and blood pressure in this systematic review. This means herbal medicines have the potential to be complementary and alternative medicines for metabolic syndrome. However, more high quality trials are needed to prove the efficacy and safety of herbal medicines.

\section{Competing Interests}

The authors declare that there are no competing interests regarding the publication of this paper.

\section{Acknowledgments}

This study was supported by a grant from the Traditional Korean Medicine R\&D Project, Ministry of Health \& Welfare, Republic of Korea (HI13C0530).

\section{References}

[1] R. H. Eckel, S. M. Grundy, and P. Z. Zimmet, "The metabolic syndrome," The Lancet, vol. 365, no. 9468, pp. 1415-1428, 2005.

[2] K. G. M. M. Alberti and P. Z. Zimmet, "Definition, diagnosis and classification of diabetes mellitus and its complications. Part 1: diagnosis and classification of diabetes mellitus. Provisional report of a WHO consultation," Diabetic Medicine, vol. 15, no. 7, pp. 539-553, 1998.

[3] S. M. Grundy, J. I. Cleeman, S. R. Daniels et al., "Diagnosis and management of the metabolic syndrome: an American Heart Association/National Heart, Lung, and Blood Institute scientific statement," Circulation, vol. 112, no. 17, pp. 2735-2752, 2005.

[4] "Third report of the National Cholesterol Education Program (NCEP) expert panel on detection, evaluation, and treatment of high blood cholesterol in adults (Adult Treatment Panel III) final report," Circulation, vol. 106, no. 25, pp. 3143-3421, 2002.

[5] D. B. Carr, K. M. Utzschneider, R. L. Hull et al., "Intraabdominal fat is a major determinant of the National Cholesterol Education Program Adult Treatment Panel III criteria for the metabolic syndrome," Diabetes, vol. 53, no. 8, pp. 2087-2094, 2004.

[6] E. Ferrannini, S. M. Haffner, B. D. Mitchell, and M. P. Stern, "Hyperinsulinaemia: the key feature of a cardiovascular and metabolic syndrome," Diabetologia, vol. 34, no. 6, pp. 416-422, 1991.

[7] J.-Q. Luo, F.-Z. He, Z.-M. Wang et al., "SLCO1B1 variants and angiotensin converting enzyme inhibitor (Enalapril)_induced cough: a pharmacogenetic study," Scientific Reports, vol. 5, Article ID 17253, 2015.

[8] S. Bangalore, S. Kumar, and F. H. Messerli, "Angiotensinconverting enzyme inhibitor associated cough: deceptive information from the physicians' desk reference," The American Journal of Medicine, vol. 123, no. 11, pp. 1016-1030, 2010.
[9] Y. Sui, H. L. Zhao, V. C. W. Wong et al., "A systematic review on use of chinese medicine and acupuncture for treatment of obesity," Obesity Reviews, vol. 13, no. 5, pp. 409-430, 2012.

[10] S. Hasani-Ranjbar, N. Nayebi, B. Larijani, and M. Abdollahi, "A systematic review of the efficacy and safety of herbal medicines used in the treatment of obesity," World Journal of Gastroenterology, vol. 15, no. 25, pp. 3073-3085, 2009.

[11] Z. Chen, L. Wang, G. Yang, H. Xu, and J. Liu, "Chinese herbal medicine combined with conventional therapy for blood pressure variability in hypertension patients: a systematic review of randomized controlled trials," Evidence-Based Complementary and Alternative Medicine, vol. 2015, Article ID 582751, 16 pages, 2015.

[12] G. Y. Yeh, D. M. Eisenberg, T. J. Kaptchuk, and R. S. Phillips, "Systematic review of herbs and dietary supplements for glycemic control in diabetes," Diabetes Care, vol. 26, no. 4, pp. 12771294, 2003.

[13] R. P. Agrawal, J. Shereyans, C. Amit Jr., and J. Ashok, "Effect of herbal medicine Dia-No on clinical and biochemical parameters of metabolic syndrome," Australian Journal of Medical Herbalism, vol. 20, no. 3, pp. 113-118, 2008.

[14] Y. Chen, D.-Y. Fu, X.-D. Fu, Y.-M. He, and W.-J. Wang, "Effect of yiqi huaju recipe combined with routine therapy in treating hypertension patients with metabolic syndrome: a clinical study," Chinese Journal of Integrated Traditional and Western Medicine, vol. 34, no. 6, pp. 680-687, 2014.

[15] H. Zhang, Z. Xie, W. Shen, S. Liao, G. Yu, and F. Huang, "Daotan Decoction in treating metabolic syndrome of phlegmdampness constitution," Jilin Journal of Traditional Chinese Medicine, vol. 34, no. 4, pp. 376-378, 2014.

[16] Y. Xu, B.-H. Hou, and B. Li, "Intervention effects of gegen shanzha decoction combining with TCM physique recuperation on metabolism syndrome," Chinese Journal of Traditional Medical Science and Technology, vol. 21, no. 4, pp. 362-364, 2014.

[17] J.-G. Li, "Clinical observation of Xuefu Zhuyu decoction in the treatment of metabolic syndrome with blood statis syndrome," Beifang Yaoxue, vol. 11, no. 11, p. 66, 2014.

[18] Y.-J. Wang, W.-F. Zhu, X.-K. Wang et al., "Study on the effect of Qinggan Jiangtang tablet in improving the insulin resistance in patients with multiple metabolic syndrome," Chinese Journal of Integrated Traditional and Western Medicine, vol. 25, no. 5, pp. 412-415, 2005.

[19] R.-D. Liu, R.-P. Huang, Y.-H. Zhang, and H.-R. Yao, "Effects of modified banxia baizhu tianma decoction on metabolic syndrome of exuberance of phlegm-dampness type," Chinese Archives of Traditional Chinese Medicine, vol. 26, no. 10, pp. 2242-2244, 2008.

[20] Y. Zhang, Y.-J. Liu, J. Zhang, Q. Li, L.-R. Chen, and B. Liu, "Effects of Pinggan Jiangya pill on blood pressure and body mass index for metabolic syndrome," Journal of Emergency in Traditional Chinese Medicine, vol. 19, no. 7, pp. 1128-1129, 2010.

[21] H.-B. Guan, X.-X. Yuan, M. Yu, and N. Xie, "Clinical observation of huanglian wendan decoction in treating metabolic syndrome," Journal of Liaoning University of TCM, vol. 14, no. 2, pp. 88-89, 2012.

[22] X. Dong, "Clinical research on xueguan ruanhua decoction in treating metabolic syndrome," China Journal of Chinese Medicine, vol. 27, no. 171, pp. 1006-1007, 2012.

[23] M. Cui and X. Wie, "Clinical observation of Shengjiangtongmai powder on the treatment of metabolic syndrome," Hubei Journal of Traditional Chinese Medicine, vol. 36, no. 1, pp. 14-16, 2014. 
[24] D.-Y. Zhao and D.-Y. Zhao, "Clinical observation of Shenling Jianpihuashi decoction in the treatment of metabolic syndrome with spleen-deficiency and stagnation of dampness," Acta Chinese Medicine and Pharmacology, vol. 43, no. 3, pp. 65-68, 2015.

[25] J. Tan, G.-D. Han, and J.-X. Teng, "Epidemiological survey on TCM syndrome distribution and season correlation of hypertension in Hunan Province," Chinese Journal of Integrated Traditional and Western Medicine, vol. 28, no. 10, pp. 897-899, 2008.

[26] Y. Nagata, H. Goto, H. Hikiami et al., "Effect of Keishibukuryogan on endothelial function in patients with at least one component of the diagnostic criteria for metabolic syndrome: a controlled clinical trial with crossover design," Evidence-Based Complementary and Alternative Medicine, vol. 2012, Article ID 359282, 10 pages, 2012.

[27] T.-Z. Wang, Y. Chen, Y.-M. He et al., "Effects of Chinese herbal medicine Yiqi Huaju Qingli Formula in metabolic syndrome patients with microalbuminuria: a randomized placebocontrolled trial," Journal of Chinese Integrative Medicine, vol. 11, no. 3, pp. 175-183, 2013.

[28] P. Ling, "Clinical observation on the effect of Ba-Wei-Wan decoction in treating patients with metabolic syndrome," Sichuan Journal of Physiological Sciences, vol. 27, no. 2, pp. 78-82, 2005.

[29] Q. Xin, Z. Hengxia, L. Zengying, and W. Taifen, "Clinical study on the treatment of metabolic syndrome by Heqi San," China Journal of Chinese Medicine, vol. 27, no. 171, pp. 1008-1010, 2012.

[30] D. Wang, Q. Hong, M. Yang et al., "Observation of Baoling decoction on elderly insulin resistance of metabolic syndrome," Hebei Journal of Traditional Chinese Medicine, vol. 34, no. 12, pp. 1779-1781, 2012.

[31] Z. Ying, "Clinical observation on the treatment of metabolic syndrome (identified as Liver Depression and Spleen Deficiency Pattern) by combination of four gentlemen decoction and sini powder," Guiding Journal of Traditional Chinese Medicine and Pharmacy, vol. 18, no. 8, pp. 29-30, 2012.

[32] M. C. Kho, Y. J. Lee, J. H. Park et al., "Combination with Red ginseng and Polygoni Multiflori ameliorates highfructose diet induced metabolic syndrome," BMC Complementary and Alternative Medicine, vol. 16, no. 1, pp. 98-108, 2016.

[33] J. Yin, H. Zhang, and J. Ye, "Traditional Chinese medicine in treatment of metabolic syndrome," Endocrine, Metabolic and Immune Disorders_Drug Targets, vol. 8, no. 2, pp. 99-111, 2008.

[34] A. Sahebkar, G. Beccuti, L. E. Simental-Mendía, V. Nobili, and S. Bo, "Nigella sativa (black seed) effects on plasma lipid concentrations in humans: a systematic review and metaanalysis of randomized placebo-controlled trials," Pharmacological Research, vol. 106, pp. 37-50, 2016.

[35] P. Tiwari, B. N. Mishra, and N. S. Sangwan, "Phytochemical and pharmacological properties of Gymnema sylvestre: an important medicinal plant," BioMed Research International, vol. 2014, Article ID 830285, 18 pages, 2014. 


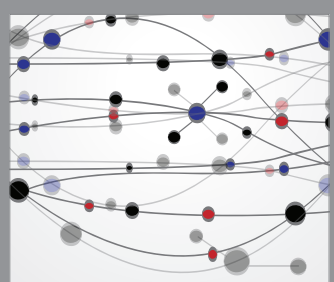

The Scientific World Journal
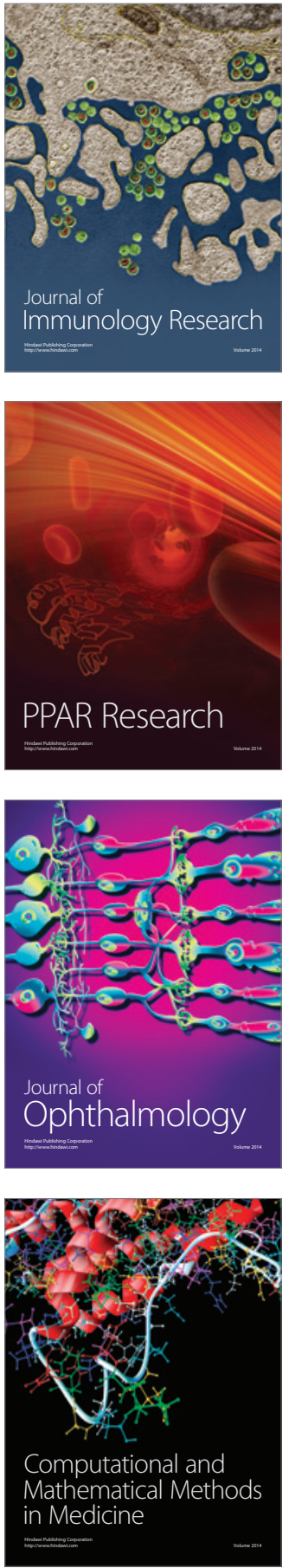

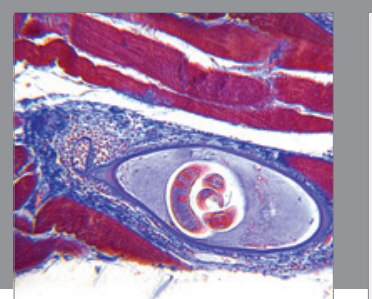

Gastroenterology Research and Practice

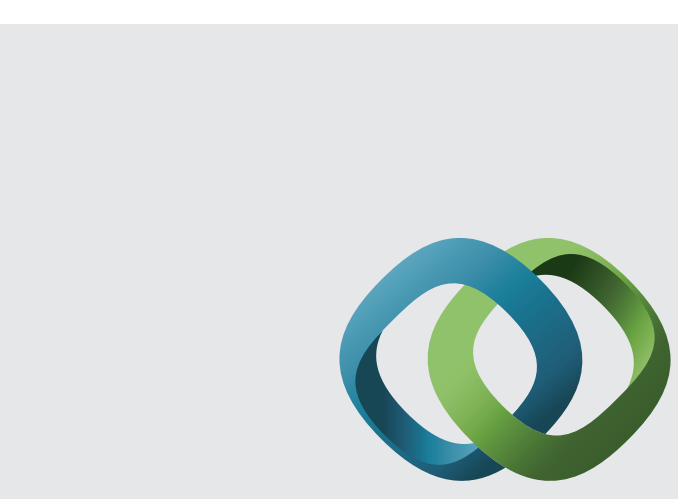

\section{Hindawi}

Submit your manuscripts at

http://www.hindawi.com
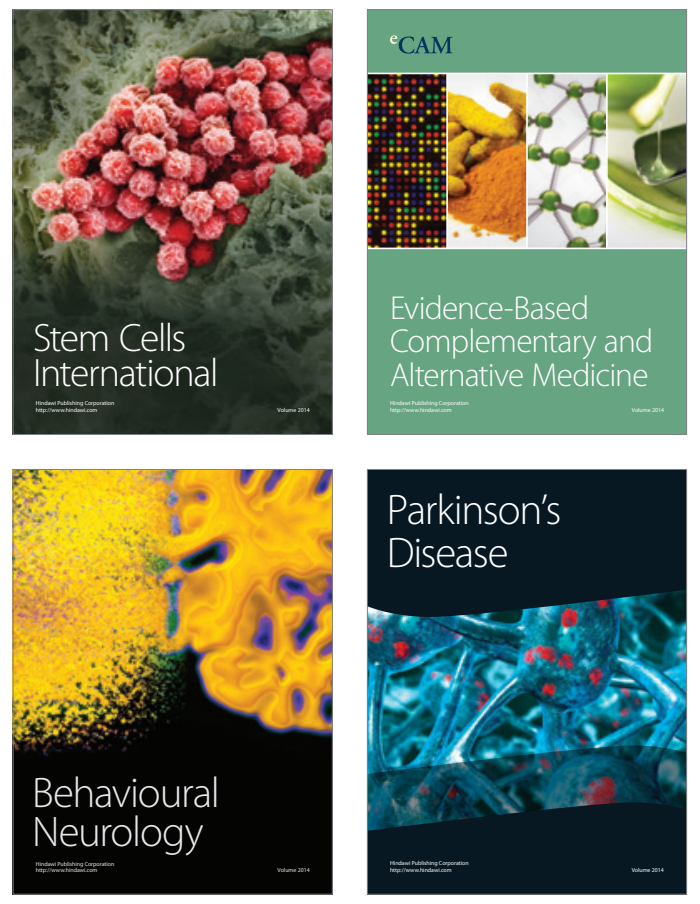
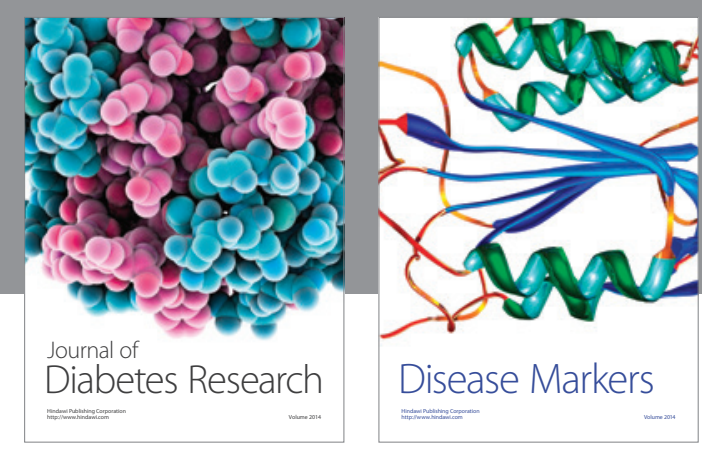

Disease Markers
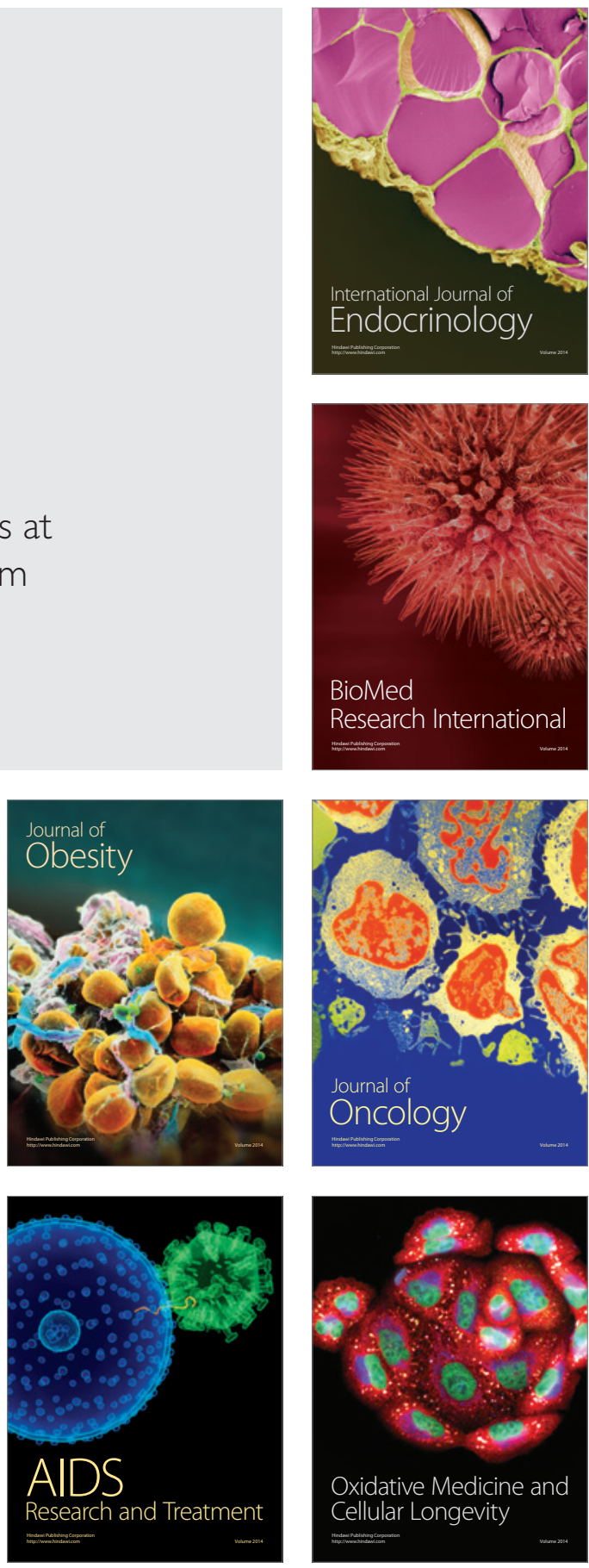